\title{
Correction to Fractional Integration and Certain Dual Integral Equations
}

Math. Zeitschr. 98, 83-88 (1967)

RoOP NARAIN KesarwaNI

In my paper the following changes should be made.

The superscript of the operator $I$ in Eq. (3.5), p. 86, should be $-c_{1}, c_{1}-a_{1}$ instead of $-c_{1}, a_{1}-c_{1}$.

The superscripts of the operators $I$ in (3.9) and (3.13), p.87, should be $-c_{i}, c_{i}-a_{i}$ instead of $-c_{i}, a_{i}-c_{i}$.

Lines 4 and 5 of Section 4, p.87 should read: "(3.12) one can change $a_{1}, \ldots, a_{n}$ and $b_{m+1}, \ldots, b_{q}$ in (1.4) respectively into $c_{1}, \ldots, c_{n}$ and $d_{m+1}, \ldots, d_{q}$, and by applying (3.10) and (3.11) one can change $c_{n+1}, \ldots, c_{p}$ and $d_{1}, \ldots, d_{m}$ in (1.5) respectively into $a_{n+1}, \ldots, a_{p}$ and $b_{1}, \ldots, b_{m}$,".

In Formula (4.1), the first and second row of parameters should be replaced respectively by:

$$
c_{1}, \ldots, c_{n}, a_{n+1}, \ldots, a_{p} \text { and } b_{1}, \ldots, b_{m}, d_{m+1}, \ldots, d_{q} .
$$

The Formula (4.2) should be replaced by:

$$
F(x)=\left\{\begin{array}{ccc}
\prod_{i=1}^{n} I_{x^{A}}^{-c_{i}, c_{i}-a_{i}} \prod_{i=m+1}^{q} I_{x^{A}}^{-b_{i}, b_{i}-d_{i}} g(x) & 0<x \leqq 1 \\
\prod_{i=1}^{m} K_{x^{A}}^{b_{i, d_{i}}-b_{i}} \prod_{i=n+1}^{p} K_{x^{A}}^{c_{i}, a_{i}-c_{i}} h(x) & 1<x<\infty \\
& \\
& \text { R. N. Kesarwani } \\
& \text { Department of Mathematics } \\
& \text { University of Ottawa } \\
& \text { Ottawa-2 (Canada) }
\end{array}\right.
$$

